\title{
Rational Trigonometric Interpolation and Constrained Control of the Interpolant Curves
}

\author{
S S Rana \\ Professor \\ Department of Mathematics, \\ R.D. University \\ Jabalpur,India
}

\author{
Mridula Dube \\ Professor \\ Department of Mathematics, \\ R.D. University \\ Jabalpur,India
}

\author{
Preeti Tiwari \\ Asst. Professor \\ Department of Mathematics \\ G.G.I.T.S. \\ Jabalpur, India
}

\begin{abstract}
In the present paper a new method is developed for smooth rational cubic trigonometric interpolation based on values of function which is being interpolated. This rational cubic trigonometric spline is used to constrain the shape of the interpolant such as to force it to be in the given region by selecting suitable parameters. The more important achievement mathematically of this method is that the uniqueness of the interpolating function for the given data would be replaced by uniqueness of the interpolating curve for the given data and selected parameters. Approximation properties have been discussed and confirms that the expected approximation order is $O\left(h^{2}\right)$.
\end{abstract}

\section{Keywords}

Rational cubic trigonometric spline, curve design, error estimation, constrained interpolation, continuity, shape parameters.

\section{INTRODUCTION}

In Computer aided design and manufacturing processes, it is usually required to generate smooth function passes through given data set. From the last decade demand for more effective tools of computer aided design is high due to increase in model complexity and manufacturing requirements in curve and surface design .Within this content, constrained design has been identified as one of the surface design problems that need to be solved. Spline interpolation has been observed as a powerful tool for curve and surface design.

Among the many generalizations of polynomial splines, the trigonometric splines are of particular theoretical interest and practical importance. The trigonometric B-splines were first introduced by Schoenberg [13]. A study of trigonometric splines has been made by a number of authors, $[1,4,5,7,10]$. It was found that problems of scattered data interpolation over spherical surfaces can be better handled in terms of accuracy, computational convenience and smoothness of the resulting surface using trigonometric splines. Trigonometric splines have been studied from application point of view in various problems of geometric modeling. Recently trigonometric splines and polynomials have gained very much interest

within CAGD in particular curve designing and in different areas such as electronics or medicine.[15]

Many authors have worked in the area of shape preservation[1,2,3,8,11]. In some manufacturing process the derivative data are not easily available, because of this we have constructed a rational cubic trigonometric spline based on the use of function values only. It is of interest that the shape of the interpolating curves can be controlled just by selection of parameters used in the construction.

The present work is organized as follows: In section 2 , construction of a rational cubic trigonometric spline with shape parameters $\alpha_{i}, \beta_{i}$ is given. section 3 , deals with the region control of the interpolant curves. The sufficient condition for constraining the interpolating curves to be bounded between two given straight lines is also derived. In section 4 , a numerical example with graphical representation is given to show how the interpolating curve can be controlled in the given region. In section 5, the approximation properties of the interpolant are discussed.

\section{A $C^{1}$ RATIONAL CUBIC TRIGONOMETRIC SPLINE INTERPOLATION}

Let $\left\{\left(t_{i}, f_{i}\right), i=0,1, \ldots, n, n+1\right\}$ be a given set of data points, such that $a=t_{0}<t_{1}<\ldots<t_{n}<t_{n+1}=b$. The $C^{1}$-continuous piecewise cubic trigonometric function based on function values is defined by

$P(t)=\frac{p_{i}(t)}{q_{i}(t)}, \quad i=0,1, \ldots, n-1$,

where

$$
\begin{aligned}
p_{i}(t)= & \left(1-\sin \frac{\pi \theta}{2}\right)^{3} \alpha_{i} f_{i}+\sin \frac{\pi \theta}{2}\left(1-\sin \frac{\pi \theta}{2}\right)\left(3-\sin \frac{\pi \theta}{2}\right) U_{i} \\
& +\cos \frac{\pi \theta}{2}\left(1-\cos \frac{\pi \theta}{2}\right)\left(3-\cos \frac{\pi \theta}{2}\right) V_{i}+\left(1-\cos \frac{\pi \theta}{2}\right)^{3} \beta_{i} f_{i+1} \\
q_{i}(t)= & \left(1-\sin \frac{\pi \theta}{2}\right)^{3} \alpha_{i}+\sin \frac{\pi \theta}{2}\left(1-\sin \frac{\pi \theta}{2}\right)\left(3-\sin \frac{\pi \theta}{2}\right) \\
& +\cos \frac{\pi \theta}{2}\left(1-\cos \frac{\pi \theta}{2}\right)\left(3-\cos \frac{\pi \theta}{2}\right)+\left(1-\cos \frac{\pi \theta}{2}\right)^{3} \beta_{i}
\end{aligned}
$$

$$
\text { with } \quad h_{i}=t_{i+1}-t_{i}, \quad \theta(t)=\frac{\left(t-t_{i}\right)}{h_{i}}, t \varepsilon\left[t_{i}, t_{i+1}\right]
$$

and 


$$
\begin{gathered}
U_{i}=f_{i}+\frac{2 h_{i} \alpha_{i}}{3 \pi} \Delta_{i} \\
V_{i}=f_{i+1}-\frac{2 h_{i} \beta_{i}}{3 \pi} \Delta_{i+1}
\end{gathered}
$$

With $\quad \alpha_{i}, \beta_{i}>0 \quad$ and $\quad \Delta_{i}=\frac{f_{i+1}-f_{i}}{h_{i}}$

This rational cubic trigonometric interpolating function satisfies

$$
P\left(t_{i}\right)=f_{i} \quad \text { and } \quad p^{\prime}\left(t_{i}\right)=\Delta_{i} .
$$

\section{SHAPE CONTROL OF THE INTERPOLATION CURVE}

For a given straight line $\mathrm{g}(\mathrm{t})$ or piecewise linear curve defined on $\left[t_{0}, t_{n}\right]$ with joints of the partition $\Delta: t_{0}<t_{1}<\ldots<t_{n}<t_{n+1}$ and a data set $\left\{\left(t_{i}, f_{i}\right): i=0,1, \ldots, n, n+1\right\}$ with

$$
f\left(t_{i}\right) \geq(\leq) g\left(t_{i}\right) \quad i=0,1, \ldots, n, n+1,
$$

let $\mathrm{P}(\mathrm{t})$ be a cubic trigonometric rational interpolating function defined by (1), when $P(t) \geq(\leq) g(t)$ for all $t \varepsilon\left[t_{0}, t_{n}\right]$,

Suppose

$$
P(t)=\frac{p_{i}(t)}{q_{i}(t)} \geq g(t),
$$

which is equivalent to

$$
p_{i}(t)-q_{i}(t) g(t)=M_{i}(t) \geq 0 .
$$

From (2) and (3) we can write (4) as

$$
\begin{aligned}
M_{i}(t)= & \left\{\left(1-\sin \frac{\pi \theta}{2}\right)^{3} \alpha_{i} f_{i}+\sin \frac{\pi \theta}{2}\left(1-\sin \frac{\pi \theta}{2}\right)\left(3-\sin \frac{\pi \theta}{2}\right) U_{i}\right. \\
& +\cos \frac{\pi \theta}{2}\left(1-\cos \frac{\pi \theta}{2}\right)\left(3-\cos \frac{\pi \theta}{2}\right) V_{i} \\
& \left.+\left(1-\cos \frac{\pi \theta}{2}\right)^{3} \beta_{i} f_{i+1}\right\}-\left\{\left(1-\sin \frac{\pi \theta}{2}\right)^{3} \alpha_{i}\right. \\
& +\sin \frac{\pi \theta}{2}\left(1-\sin \frac{\pi \theta}{2}\right)\left(3-\sin \frac{\pi \theta}{2}\right)+\cos \frac{\pi \theta}{2}\left(1-\cos \frac{\pi \theta}{2}\right)\left(3-\cos \frac{\pi \theta}{2}\right) \\
& \left.+\left(1-\cos \frac{\pi \theta}{2}\right)^{3} \beta_{i}\right\}\left[(1-\theta) g_{i}+\theta g_{i+1}\right] \geq 0
\end{aligned}
$$

where $g_{i}, g_{i+1}$ represent $g\left(t_{i}\right), g\left(t_{i+1}\right)$, respectively. Since

$$
\begin{aligned}
M_{i}(t)= & \left(1-\sin \frac{\pi \theta}{2}\right)^{3} \alpha_{i} A_{i}+\sin \frac{\pi \theta}{2}\left(1-\sin \frac{\pi \theta}{2}\right)\left(3-\sin \frac{\pi \theta}{2}\right) B_{i} \\
& +\cos \frac{\pi \theta}{2}\left(1-\cos \frac{\pi \theta}{2}\right)\left(3-\cos \frac{\pi \theta}{2}\right) C_{i}+\left(1-\cos \frac{\pi \theta}{2}\right)^{3} \beta_{i} D_{i} \geq 0
\end{aligned}
$$

where

$$
\begin{aligned}
A_{i} & =(1-\theta)\left(f_{i}-g_{i}\right)+\theta\left(f_{i+1}-g_{i+1}\right)-\theta h_{i} \Delta_{i} \\
B_{i} & =A_{i}+\frac{2 \alpha_{i} h_{i}}{3 \pi} \Delta_{i} \\
C_{i} & =D_{i}-\frac{2 h_{i} \beta_{i}}{3 \pi} \Delta_{i+1} \\
D_{i} & =A_{i}+h_{i} \Delta_{i}
\end{aligned}
$$

for $\alpha_{i}, \beta_{i}>0$,and Since $f_{i}-g_{i} \geq 0$, for all i, we find a sufficient condition for the rational cubic trigonometric curve $P(t)$ to lie above the straight line $g(t)$ in $\left[t_{i}, t_{i+1}\right]$ given in the following theorem:

Theorem 3.1Given $\left\{\left(t_{i}, f_{i}, g_{i}\right), \quad i=0,1, \ldots, n, n+1\right\}$ with $f_{i} \geq g_{i}, i=0,1, \ldots, n$, the sufficient condition for the rational cubic trigonometric curve $P(t)$ defined by (1) to lie above the straight line $g(t)$ in $\left[t_{i}, t_{i+1}\right]$ is that the positive parameters $\alpha_{i}, \beta_{i}$, satisfy

$$
\begin{aligned}
& A_{i} \geq \mathrm{O} \\
& \boldsymbol{A}_{i}+\frac{2 \alpha_{i} \boldsymbol{h}_{i}}{3 \pi} \Delta_{i}>\mathrm{O} \\
& D_{i} \geq \mathrm{O} \\
& D_{i}-\frac{2 \boldsymbol{h}_{i} \boldsymbol{\beta}_{i}}{3 \pi} \Delta_{i+1} \geq \mathrm{O}
\end{aligned}
$$

For equally spaced partition Theorem 3.1 has the following corollary:

Corollary 3.1 For the equally spaced partition, the sufficient condition for the rational cubic trigonometric curve $P(t)$ defined by (1) to lie above the straight line $g(t)$ in $\left[t_{i}, t_{i+1}\right]$ is that the positive parameters $\alpha_{i}, \beta_{i}$ satisfy the conditions

$$
\begin{aligned}
& (1-\theta)\left(f_{i}-g_{i}\right)+\theta\left(f_{i+1}-g_{i+1}\right)-\theta\left(f_{i+1}-f_{i}\right) \geq 0 \\
& (1-\theta)\left(f_{i}-g_{i}\right)+\theta\left(f_{i+1}-g_{i+1}\right)-\theta\left(f_{i+1}-f_{i}\right)+\frac{2 \alpha_{i}}{3 \pi}\left(f_{i+1}-f_{i}\right) \geq 0 \\
& (1-\theta)\left(f_{i}-g_{i}\right)+\theta\left(f_{i+1}-g_{i+1}\right)+(1-\theta)\left(f_{i+1}-f_{i}\right)-\frac{2 h_{i} \beta_{i}}{3 \pi}\left(f_{i+2}-f_{i+1}\right) \geq 0 \\
& (1-\theta)\left(f_{i}-g_{i}\right)+\theta\left(f_{i+1}-g_{i+1}\right)+(1-\theta)\left(f_{i+1}-f_{i}\right) \geq 0
\end{aligned}
$$

Theorem 3.2 Given $\left\{\left(t_{i}, f_{i}, g_{i}\right), i=0,1, \ldots, n, n+1\right\}$ with $\quad$ g_i $f_{i} \leq g^{*}{ }_{i}, i=0,1, \ldots, n+1, \quad$ the sufficient condition for the rational cubic trigonometric curve $P(t)$ defined by (1) to lie above the straight line $g(t)$ and below the straight line $g^{*}(t)$ in $\left[t_{i}, t_{i+1}\right]$ is that the positive parameters $\alpha_{i}, \beta_{i}$, satisfy 


$$
\begin{aligned}
& (1-\theta)\left(f_{i}-g_{i}\right)+\theta\left(f_{i+1}-g_{i+1}\right)-\theta h_{i} \Delta_{i} \geq 0 \\
& (1-\theta)\left(f_{i}-g_{i}\right)+\theta\left(f_{i+1}-g_{i+1}\right)-\theta h_{i} \Delta_{i}+\frac{2 \alpha_{i} h_{i}}{3 \pi} \Delta_{i} \geq 0 \\
& (1-\theta)\left(f_{i}-g_{i}\right)+\theta\left(f_{i+1}-g_{i+1}\right)-\theta h_{i} \Delta_{i}+h_{i} \Delta_{i}-\frac{2 h_{i} \beta_{i}}{3 \pi} \Delta_{i+1} \geq 0 \\
& (1-\theta)\left(f_{i}-g_{i}\right)+\theta\left(f_{i+1}-g_{i+1}\right)-\theta h_{i} \Delta_{i}+h_{i} \Delta_{i} \geq 0
\end{aligned}
$$

and

$$
\begin{aligned}
& (1-\theta)\left(g_{i}{ }^{*}-f_{i}\right)+\theta\left(g_{i+1}{ }^{*}-f_{i+1}\right)+\theta h_{i} \Delta_{i} \geq 0 \\
& (1-\theta)\left(g_{i}{ }^{*}-f_{i}\right)+\theta\left(g_{i+1}{ }^{*}-f_{i+1}\right)+\theta h_{i} \Delta_{i}-\frac{2 \alpha_{i} h_{i}}{3 \pi} \Delta_{i} \geq 0 \\
& (1-\theta)\left(g_{i}{ }^{*}-f_{i}\right)+\theta\left(g_{i+1}{ }^{*}-f_{i+1}\right)+\theta h_{i} \Delta_{i}+h_{i} \Delta_{i}+\frac{2 h_{i} \beta_{i}}{3 \pi} \Delta_{i+1} \geq 0 \\
& (1-\theta)\left(g_{i}{ }^{*}-f_{i}\right)+\theta\left(g_{i+1}{ }^{*}-f_{i+1}\right)+\theta h_{i} \Delta_{i}+h_{i} \Delta_{i} \geq 0
\end{aligned}
$$

\section{Numerical Example}

The interpolating and constraining data are given in Table 1 and the parameters and $\alpha_{i}$ and $\beta_{i} \quad(i=1,2,3)$ are given in Table 2 . It is to test that both the given interpolating and constraining data and the parameters satisfy the relationship (4) and the constraint inequalities (7), (8) so the interpolating curve $P(t)$ defined by (1) must be bounded between $g^{*}(t)$ and $g(t)$, which can be written as

$$
g^{*}(t)= \begin{cases}\frac{-8}{50} t+0.107 & , 0 \leq t \leq 0.5 \\ \frac{6}{50} t-0.033 & , 0.5 \leq t \leq 1.0 \\ \frac{-4}{50} t+0.167 & , 1.0 \leq t \leq 1.5\end{cases}
$$

$$
g(t)= \begin{cases}-\frac{8}{50} t+0.093 & , 0 \leq t \leq 0.5 \\ \frac{6}{50} t-0.047 & , 0.5 \leq t \leq 1.0 \\ -\frac{4}{50} t+0.153 & , 1.0 \leq t \leq 1.5\end{cases}
$$

Fig. 1 shows that the interpolating curve $P(t)$ is bounded between $g^{*}(t)$ and $g(t)$.

Table 1 The interpolating data and the constraining data

\begin{tabular}{|c|c|c|c|c|}
\hline $\mathbf{t}_{\mathbf{i}}$ & $\mathbf{i}$ & $\mathbf{g * ( \mathbf { t } _ { \mathbf { i } } )}$ & $\mathbf{f}\left(\mathbf{t}_{\mathbf{i}}\right)$ & $\mathbf{g}\left(\mathbf{t}_{\mathbf{i}}\right)$ \\
\hline 0.0 & 1 & 0.107 & 0.1 & 0.093 \\
\hline 0.5 & 2 & 0.027 & 0.02 & 0.013 \\
\hline 1.0 & 3 & 0.087 & 0.08 & 0.073 \\
\hline 1.5 & 4 & 0.047 & 0.04 & 0.033 \\
\hline 2.0 & 5 & 0.067 & 0.06 & 0.053 \\
\hline
\end{tabular}

Table 2 The parameters $\alpha_{i}$ and $\beta_{i}$ for rational trigonometric interpolation

\begin{tabular}{|c|c|c|}
\hline $\mathbf{i}$ & $\alpha_{i}$ & $\beta_{i}$ \\
\hline 1 & 0.001123 & 0.0011423 \\
\hline 2 & 0.001555 & 0.00124 \\
\hline 3 & 0.001510 & 0.0011905 \\
\hline
\end{tabular}

$$
R[f]=f(t)-P(t)=\int_{t_{i}}^{t_{i+1}} f^{(2)}(\tau) R_{t}\left[(t-\tau)_{+}\right] \mathrm{d} \tau,
$$$$
t \in\left[t_{i}, t_{i+1}\right]
$$

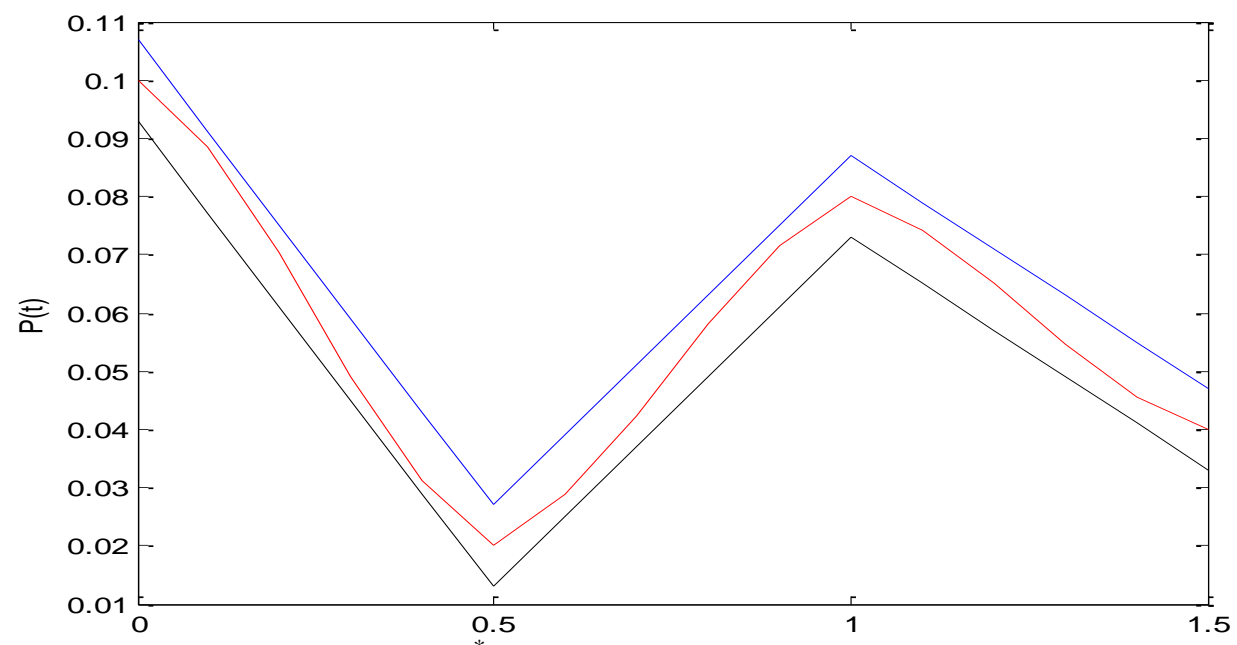

Figure 1: graph of $g^{*}(t), P(t)$ and $g(t)$ for data in Table 1 and 2 . 


\section{APPROXIMATION PROPERTIES OF THE DEFINED INTERPOLATION}

To estimate error of the rational trigonometric interpolating function defined by (1), since the interpolation is local, without loss of generality, consider the error in the subinterval $\left[t_{i}, t_{i+1}\right]$. When $f(t) \varepsilon C^{1}\left[t_{0}, t_{n}\right]$ and $\mathrm{P}(\mathrm{t})$ is the rational trigonometric spline interpolating function of $f(t)$ in $\left[t_{i}, t_{i+1}\right]$. It is easy to see that this type of interpolation is exact for $f(t)$, the polynomial being interpolated,in which the degree is no more than 1 . Consider the case when the knots are equally spaced,namely, $h_{i}=h=\frac{t_{n}-t_{0}}{n}$ for all $i=1,2, \ldots, n$, using the Peano-Kernel Theorem in Schultz [14] gives the following

where

$$
R_{t}\left[(t-\tau)_{+}\right]=\left\{\begin{array}{c}
p(\tau) \quad t_{i}<\tau<t \\
q(\tau) \quad t<\tau<t_{i+1} \\
r(\tau) \quad t_{i+1}<\tau<t_{i+2}
\end{array}\right.
$$

where

$$
\begin{aligned}
& p(\tau)=(t-\tau)-\left\{\left[( t _ { i + 1 } - \tau ) \left[\sin \frac{\pi \theta}{2}\left(1-\sin \frac{\pi \theta}{2}\right)\left(3-\sin \frac{\pi \theta}{2}\right) \frac{2 \alpha_{i}}{3 \pi}\right.\right.\right. \\
& \left.+\cos \frac{\pi \theta}{2}\left(1-\cos \frac{\pi \theta}{2}\right)\left(3-\cos \frac{\pi \theta}{2}\right)+\left(1-\cos \frac{\pi \theta}{2}\right)^{3} \beta_{i}\right] \\
& \left.\left.-\frac{2 h_{i} \beta_{i}}{3 \pi} \cos \frac{\pi \theta}{2}\left(3-\cos \frac{\pi \theta}{2}\right)\right] / N\right\} \quad t_{i}<\tau<t ; \\
& q(\tau)=-\left\{\left[( t _ { i + 1 } - \tau ) \left[\sin \frac{\pi \theta}{2}\left(1-\sin \frac{\pi \theta}{2}\right)\left(3-\sin \frac{\pi \theta}{2}\right) \frac{2 \alpha_{i}}{3 \pi}\right.\right.\right. \\
& \left.+\cos \frac{\pi \theta}{2}\left(1-\cos \frac{\pi \theta}{2}\right)\left(3-\cos \frac{\pi \theta}{2}\right)+\left(1-\cos \frac{\pi \theta}{2}\right)^{3} \beta_{i}\right] \\
& \left.\left.-\frac{2 h_{i} \beta_{i}}{3 \pi} \cos \frac{\pi \theta}{2}\left(1-\cos \frac{\pi \theta}{2}\right)\left(3-\cos \frac{\pi \theta}{2}\right)\right] / N\right\} \\
& t_{i}<\tau<t \text {; }
\end{aligned}
$$

$$
\begin{gathered}
r(\tau)=\left\{\left[\frac{\left(2 \beta_{i}\right)}{3 \pi} \cos \frac{\pi \theta}{2}\left(1-\cos \frac{\pi \theta}{2}\right)\left(3-\cos \frac{\pi \theta}{2}\right)\left(t_{i+2}-\tau\right)\right] / N\right\} \\
t_{i+1}<\tau<t_{i+2}
\end{gathered}
$$

where

$$
\begin{aligned}
N=(1 & \left.-\sin \frac{\pi \theta}{2}\right)^{3} \alpha_{i}+\sin \frac{\pi \theta}{2}\left(1-\sin \frac{\pi \theta}{2}\right)\left(3-\sin \frac{\pi \theta}{2}\right) \\
& \left.\left.+\cos \frac{\pi \theta}{2}\left(1-\cos \frac{\pi \theta}{2}\right)\left(3-\cos \frac{\pi \theta}{2}\right)+\left(1-\cos \frac{\pi \theta}{2}\right)^{3} \beta_{i}\right]\right\}
\end{aligned}
$$

Then

$$
\begin{aligned}
& \square R[f] \square=\square f(t)-P(t) \square \\
& \leq \square f^{2}(t) \square\left[\int_{t_{i}}^{t}|p(\tau)| \mathrm{d} \tau+\int_{t}^{t_{i+1}}|q(\tau)| \mathrm{d} \tau+\int_{t_{i+1}}^{t_{i+2}}|r(\tau)| \mathrm{d} \tau\right]
\end{aligned}
$$

for $\lambda \leq 1 \quad r(\tau) \geq 0$ for all $\tau \varepsilon\left[t_{i+1}, t_{i+2}\right]$ it may be seen that

$$
\int_{t_{i+1}}^{t_{i+2}}|r(\tau)| \mathrm{d} \tau=h^{2} W_{1}
$$

where

$W_{1}=\frac{1}{2}\left\{\frac{\frac{2 \beta_{i}}{3 \pi} \cos \frac{\pi \theta}{2}\left(1-\cos \frac{\pi \theta}{2}\right)\left(3-\cos \frac{\pi \theta}{2}\right)}{M}\right\}$

with

$$
\begin{aligned}
M= & \frac{2 \alpha_{i}}{3 \pi} \sin \frac{\pi \theta}{2}\left(1-\sin \frac{\pi \theta}{2}\right)\left(3-\sin \frac{\pi \theta}{2}\right) \\
& +\cos \frac{\pi \theta}{2}\left(1-\cos \frac{\pi \theta}{2}\right)\left(3-\cos \frac{\pi \theta}{2}\right)+\left(1-\cos \frac{\pi \theta}{2}\right)^{3} \beta_{i}
\end{aligned}
$$

for $q(\tau)$, since

$$
q\left(t_{i+1}\right)=\frac{\frac{2 h_{i} \beta_{i}}{3 \pi} \cos \frac{\pi \theta}{2}\left(1-\cos \frac{\pi \theta}{2}\right)\left(3-\cos \frac{\pi \theta}{2}\right)}{N} \geq 0
$$

$q(t)=-h \frac{(1-\theta) M-\frac{2 \beta_{i}}{3 \pi} \cos \frac{\pi \theta}{2}\left(1-\cos \frac{\pi \theta}{2}\right)\left(3-\cos \frac{\pi \theta}{2}\right)}{N} \leq 0$

there is a zero point $\tau^{*}$ of $\mathrm{q}(\tau)$ in $\left[t_{i}, t_{i+1}\right]$ given by

$\tau^{*}=t_{i+1}-\frac{\frac{2 h_{i} \beta_{i}}{3 \pi} \cos \frac{\pi \theta}{2}\left(1-\cos \frac{\pi \theta}{2}\right)\left(3-\cos \frac{\pi \theta}{2}\right)}{M}$

$$
\begin{aligned}
& \int_{t}^{t_{i+1}}|q(\tau)| \mathrm{d} \tau=\int_{t}^{\tau^{*}}-q(\tau) \mathrm{d} \tau+\int_{\tau^{*}}^{t_{i+1}} q(\tau) \mathrm{d} \tau \\
& =h^{2}\left[W_{2}\right]
\end{aligned}
$$

where $W_{2}=w_{1}+w_{2}$

with

$w_{1}=M\left\{\frac{(\theta-1)^{2}}{2}-\left[\frac{\frac{2 \beta_{i}}{3 \pi} \cos \frac{\pi \theta}{2}\left(1-\cos \frac{\pi \theta}{2}\right)\left(3-\cos \frac{\pi \theta}{2}\right)}{M}\right]^{2}\right\}$ 


$$
\begin{gathered}
w_{2}=\frac{2 \beta_{i}}{3 \pi} \cos \frac{\pi \theta}{2}\left(1-\cos \frac{\pi \theta}{2}\right)\left(3-\cos \frac{\pi \theta}{2}\right)[(\theta-1) \\
\left.+\frac{2 \frac{2 \beta_{i}}{3 \pi} \cos \frac{\pi \theta}{2}\left(1-\cos \frac{\pi \theta}{2}\right)\left(3-\cos \frac{\pi \theta}{2}\right)}{M}\right]
\end{gathered}
$$

Since $p(\tau)=(t-\tau)+q(\tau)$

and $\quad p\left(t_{i}\right)=\left(t-t_{i}\right)+q\left(t_{i}\right)=S \geq 0$

where

$$
S=\frac{L}{N}
$$

with

$$
\begin{aligned}
L= & h\left\{\theta\left(1-\sin \frac{\pi \theta}{2}\right)^{3} \alpha_{i}+\sin \frac{\pi \theta}{2}\left(1-\sin \frac{\pi \theta}{2}\right)\left(3-\sin \frac{\pi \theta}{2}\right)\left[\theta-\frac{2 \alpha_{i}}{3 \pi}\right]\right. \\
& +\cos \frac{\pi \theta}{2}\left(1-\cos \frac{\pi \theta}{2}\right) \quad\left(3-\cos \frac{\pi \theta}{2}\right)\left[\theta-1+\frac{2 \beta_{i}}{3 \pi}\right] \\
& +\left(1-\cos \frac{\pi \theta}{2}\right)^{3} \beta_{i}[\theta-1]
\end{aligned}
$$

$p(t)=q(t) \leq 0$ and the root $\tau_{*}$ of $p(\tau)$ in $\left[t_{i}, t\right]$ is

$$
\begin{gathered}
\tau_{*}=t_{i+1}-h \frac{(\theta-1)+\frac{2 \beta_{i}}{3 \pi} \cos \frac{\pi \theta}{2}\left(1-\cos \frac{\pi \theta}{2}\right)\left(3-\cos \frac{\pi \theta}{2}\right)}{M-1} \\
\int_{t_{i}}^{t}|p(\tau)| \mathrm{d} \tau=\int_{t_{i}}^{\tau_{*}} p(\tau) \mathrm{d} \tau+\int_{\tau_{*}}^{t}-p(\tau) \mathrm{d} \tau \\
=h^{2} W_{3}
\end{gathered}
$$

where

$W_{3}=\left[X_{1}+\frac{M}{N} X_{2}+\frac{2 \beta_{i}}{3 \pi N} \cos \frac{\pi \theta}{2}\left(1-\cos \frac{\pi \theta}{2}\right)\left(3-\cos \frac{\pi \theta}{2}\right) X_{3}\right.$

with

$$
\begin{aligned}
X_{1}=\{ & \left.\frac{\theta^{2}}{2}-\left[(1-\theta)-\frac{(\theta-1)+\frac{2 \beta_{i}}{3 \pi} \cos \frac{\pi \theta}{2}\left(1-\cos \frac{\pi \theta}{2}\right)\left(3-\cos \frac{\pi \theta}{2}\right)}{M-1}\right]^{2}\right\} \\
X_{2}=\{ & {\left[\frac{(\theta-1)+\frac{2 \beta_{i}}{3 \pi} \cos \frac{\pi \theta}{2}\left(1-\cos \frac{\pi \theta}{2}\right)\left(3-\cos \frac{\pi \theta}{2}\right)}{M-1}\right]^{2} } \\
& \left.-\frac{1}{2}\left((\theta-1)^{2}+1\right)\right\} \\
X_{3}= & \left\{2\left[1-\frac{\theta-1+\frac{2 \beta_{i}}{3 \pi} \cos \frac{\pi \theta}{2}\left(1-\cos \frac{\pi \theta}{2}\right)\left(3-\cos \frac{\pi \theta}{2}\right)}{M-1}\right]-\theta\right\}
\end{aligned}
$$

Thus,

$$
\begin{aligned}
\square R[f] \square= & \square f(t)-P(t) \square \\
& \leq \square f^{2}(t) \square\left[\int_{t_{i}}^{t}|p(\tau)| \mathrm{d} \tau+\int_{t}^{t_{i+1}}|q(\tau)| \mathrm{d} \tau+\int_{t_{i+1}}^{t_{i+2}}|r(\tau)| \mathrm{d} \tau\right] \\
& =h^{2} \square f^{2}(t) \square\left[W_{1}+W_{2}+W_{3}\right]
\end{aligned}
$$

\section{CONCLUSION}

The construction of a rational cubic trigonometric spline with shape parameters $\alpha_{i}, \beta_{i}$ is given.. The sufficient conditions for constraining the interpolating curves to be bounded between two given straight lines is derived and the approximation properties of the interpolant has been discussed. This concept may have an extensive application in surface generation

\section{ACKNOWLEDGEMENT}

The authors would like to thank referees of this paper for their valuable suggestions.

\section{REFERENCES}

[1] Abbas M, Majid A. A. ,Ali J. M., Positivity preserving interpolation of positive data by cubic trigonometric spline, Mathematika, 2011, 27, 41-50.

[2] Duan Q, Wang L, Twizell E.H., A new weighted rational cubic interpolation and its approximation, Appl. Math. Comput., 2005, 168, 990-1003.

[3] Duan Q, Wang L, Twizell EH., A new $C^{2}$ rational interpolation based on function values and constrained control of the interpolant curves, Appl. Math. Comput. 2005, 161, 311-322.

[4] Dube M, Sharma R., Quadratic NUAT B-spline curves with multiple shape parameters, International Journal of Machine Intelligence, 2011, 3, 18-24.

[5] Dube M, Tiwari P., Convexity preserving $C^{2}$ rational quadratic trigonometric spline, AIP conference proceedings of ICNAAM, 2012, 1479, 995-998.

[6] Gregory J.A., Shape preserving spline interpolation, Comput. Aided Des., 1986, 18, 53-57.

[7] Han Xi-An, Ma-YiChen, Huang XiLi., The cubic trigonometric Bézier curve with two shape parameters., Applied Mathematics Letters, 2009, 22, 226-231.

[8] Han Xuli., Quadratic trigonometric polynomial curves with a shape parameter, Computer Aided Geometric Design, 2002, 19(7), 503-512.

[9] Passow E., Roulier J.A., Monotone and convex spline interpolation, SIAM Journal of Num. Analysis, 1977, 14, 904-909.

[10] Rana S.S., Dube M, Sanyal S., A $G C^{2}$ cubic trigonometric B-spline, Investigation in Mathematical Sciences, 2011, 1, 25-31.

[11] Sarfraz M., Convexity preserving piecewise rational interpolation for planer curves, Bulletin of Korean Mathematical Society, 1999, 29, 193-200.

[12]Sarfraz M., Rational spline interpolation preserving the shape of the monotonic data. in: 'Proceeding of the Computer Graphics International', IEEE Computer Society, 1997, 97, 238-244.

[13] Schoenberg I.J., On trigonometric spline interpolation, J.Math. Mech., 1964, 13, 795-825.

[14]Schultz M.H., Spline Analysis,Prentice-Hall:Englewood Cliffs, New Jersey, 1973.

[15] Schumaker L. L., On shape preserving quadratic spline interpolation, SIAM J.Numer.Anal., 1983, 20, 854-864. 